\title{
The role of adhesion molecules in inflammatory bowel disease in children. Assessment of the possible risk of cardiovascular complications
}

\author{
Aneta Trzeciak-Jędrzejczyk ${ }^{1}$, Ryszard Makosiej ${ }^{1}$, Michał Kolejwa ${ }^{1}$, Ewa Głowacka², Elżbieta Czkwianianc ${ }^{1}$ \\ 1Department of Gastroenterology, Allergology and Pediatrics, Polish Mother's Memorial Hospital Research Institute, Lodz, Poland \\ 2Laboratory Diagnostics Centre, Polish Mother's Memorial Hospital Research Institute, Lodz, Poland
}

Gastroenterology Rev 2017; 12 (3): 181-185

DOI: https://doi.org/10.5114/pg.2017.70480

Key words: adhesion molecules, inflammatory bowel diseases, atherosclerosis, children.

Address for correspondence: Aneta Trzeciak-Jędrzejczyk MD, PhD, Department of Gastroenterology, Allergology and Pediatrics, Polish Mother's Memorial Hospital Research Institute, 281/289 Rzgowska St, 93-338 Lodz, Poland, phone: +48 42271 13 41, fax: +48 42271 13-40, e-mail: jdamian29@interia.pl

\begin{abstract}
Introduction: Inflammatory bowel diseases (IBD) are chronic diseases that proceed with exacerbation and remission phases. Adhesion molecules play a significant role in inflammatory processes. The same adhesion molecules play an important role in atherogenesis.

Aim: To assess the risk of atherosclerosis in IBD in children.

Material and methods: The study included 40 patients with IBD (25 with Crohn's disease - CD and 15 with ulcerative colitis - UC) aged 4-17 years. In the study group, concentrations of selected adhesion molecules (intracellular adhesion molecule ICAM, vascular cell adhesion molecule - VCAM, E-selectin) and selected parameters of lipid metabolism in serum were assessed.

Results: No statistically significant differences between CD and UC patients and in the control group, in mean values of selected adhesins were obtained. Average variable VCAM was significantly lower in patients with CD than in patients with UC in the active stage of the diseases. Significantly higher average levels of triglycerides (TG) and high density lipoproteins (HDL) were found in the control group than in patients with CD. Significantly higher levels of total cholesterol (CHL) and HDL were noticed in the control group patients than in the patients with UC. The HDL/CHL was significantly higher in controls than in patients with UC.

Conclusions: No increased risk of developing atherosclerosis was found in children with IBD. Decreased risk in patients during exacerbation of inflammatory bowel disease was revealed, which may result from malnutrition typical for acute disease phase.
\end{abstract}

\section{Introduction}

Inflammatory bowel diseases (IBD) are diseases characterised by a chronic inflammatory process, which runs with periods of exacerbation and remission. The inflammatory nature of Crohn's disease (CD) and ulcerative colitis (UC) justifies the analysis of the role of adhesion molecules in the pathogenesis of these diseases as well as their potential consequences for the vascular lesions and the development of circulatory diseases. Adhesion molecules (cell adhesion molecule - CAM) are glycoproteins involved in the embryogenesis, neoplastic, and inflammatory processes. They also participate in the attachment of leukocytes to endothelial cells, which facilitates their penetration to the inflammation area. The CAM may exist in two forms: as a transmembrane protein (mCAM) associated with the cell structures, and as a soluble protein (sCAM), which are devoid of the transmembrane and intracellular domains [1]. Currently over a hundred different adhesion molecules are known. On the basis of differences in molecular structure and their functions, we may distinguish major groups of them: selectins, integrins, cadherins, immunoglobulin-like molecules, and cell differentiation antigens [2].

In IBD various stages of inflammation process and a role of adhesions cannot be ignored. The first step in the inflammatory process is called rolling of leukocytes. The intercellular adhesion molecule (ICAM) optimises this process. As a result of rolling, migrating leukocytes approach endothelial cells, where the selectin receptors are presented. Then it comes to bonding on the surface 
of the endothelium. Selectins and vascular cell adhesion molecules (VCAM), which are responsible for the adhesion of leukocytes to the endothelium, mediate in this mechanism. Endothelial selectin plays a major role in chemotaxis of leukocytes to the vascular wall and their adhesion to the endothelium. The next step is activation of white blood cells. In the place of inflammation proinflammatory cytokines are released, which activates the integrins and contributes to slowing the flow of cells. The third stage is adhesion. Activated adhesion molecules from the integrins group provide communication between the leukocytes and endothelial cells. The final step of the inflammatory process consists of diapedesis and migration of leukocytes through the wall of blood vessels [3].

There are described attempts of assessment of disease activity degree by estimating the concentration of circulating forms of adhesion molecule and establishing correlations between these processes. In addition, it is well known that adhesion molecules play an important role in the pathogenesis of atherosclerosis.

The process of atherosclerosis begins in childhood. Its development depends on genetic and coexisting environmental factors [4-7]. Many risk factors, like a hyperlipidaemia, obesity, low physical activity, high blood pressure, diabetes, smoking, and chronic stress impacts on developing the atherosclerosis. In the early stage of atherosclerosis vascular endothelial functions are impaired. There are reports that have revealed that in patients with diagnosed structural atherosclerotic lesions (increased thickness of the intima-media carotid artery) significantly elevated lipid profile was observed in childhood [8].

Adhesions take part in reparation processes by the influence on migration of leukocytes to the inflammation. On the other hand, marking them may constitute a serum marker of inflammation of the blood vessels. The process of vessels damage by the inflammatory molecules and leucocytes might be the trigger of the cascade process for atherosclerosis initiation. The main stage in arteriosclerosis is adhesion of leukocytes to the epithelial cells. It is mediated at first by selectins and next by ICAM and VCAM. The presence of soluble forms of the adhesion molecules in the blood is considered to be the earliest stage of the atherosclerotic process [9] Detection of these substances can be a specific marker of endothelial cell activation and may provide the onset of the inflammatory process, which for the blood vessels is the basis of the further development of atherosclerosis. Potentially higher levels of ICAM and VCAM in IBD patients may indicate activation or damage of the vascular endothelium, and enable migration of inflammatory cells. Stopping and mastering this process is one of the goals in modern IBD therapy. Increasing concen- trations of these molecules in the serum can affect the inflammation and triggering of the early atherosclerotic process in patients with IBD. On the other hand, people with these diseases tend to have shorter or longer (often chronic) conditions of malnutrition, which contradicts the etiopathogenesis of atherosclerosis. There are studies that indicate that patients with IBD have even lower risk of developing atherosclerosis [10].

\section{Aim}

The aim of this study was to mark selected adhesion molecules in the serum of children with IBD and to assess the risk of developing atherosclerosis, as well as to investigate the relationship between these processes.

\section{Material and methods}

To the study 40 patients with IBD, hospitalised in the Department of Gastroenterology, Allergology, and Paediatrics, Polish Mother's Memorial Hospital Research Institute in Lodz were enrolled. Among them 25 were diagnosed with $C D$ and 15 with UC. Patients were aged between 4 and 17 years. In the group of patients with CD, 16 were in a stage of exacerbation (8 female, 8 male) and 9 in remission (4 female, 5 male). Whereas in the group of patients with CU 10 were in a stage of exacerbation (4 female, 6 male) and 5 in remission ( 2 female, 3 male). The control group constituted 21 patients (12 female, 9 male) without any symptoms of disease from the gastrointestinal tract. The average age of patients with CD was 14.1 years, median 15.0, while for patients with UC it was 13.3 years, median 14.0, and the average age of control group was 10.9 years, median 10.0.

In all patients levels of chosen adhesion molecules (ICAM, VCAM, E-selectin) in serum were measured by immunoenzymatic method. ELISA test kits from R\&D Systems, Inc., USA were used in this study. In all patients selected lipid parameters (total cholesterol, TG, $\mathrm{HDL}$, LDL, and HDL/cholesterol ratio) were marked by commercial assays.

The Bioethics Committee operating at the Institute of Polish Mother Health Centre in Lodz approved the study (Resolution No. 37/2012, 6 June 2012). Parents and children were informed about the character of the research. Parents expressed written consent to the study, while children express consent before testing.

\section{Statistical analysis}

Measures of descriptive statistics arithmetic mean and median were used. In the analysis of the studied adhesion molecules and selected lipids, arithmetic mean and standard deviation were used. Verification 
of the distribution of variables was made by the Shapiro-Wilk test. Because most investigated values deviate significantly from the normal distribution a non-parametric test was used. The statistical analysis between the study group and control group and a comparison of subgroups was performed using the Mann-Whitney test. The confidence level of $p<0.05$ was considered statistically significant.

\section{Results}

There was no statistically significant difference between patients with CD and UC $(p>0.05)$ in terms of the average values of concentration of sICAM, sVCAM, and sE-selectin. Also, no statistically significant results were obtained in comparison to the control group. The average concentrations of selected adhesion molecules in various phases of disease were additionally compared. A statistically significant difference was found in the remission between the average values obtained for VCAM concentrations in CD and UC $(p<0.05)$. In this phase the average variable VCAM was significantly lower in CD than in UC patients: $762.9 \pm 189.9 \mathrm{ng} / \mathrm{ml}$ vs $942.5 \pm 225.1 \mathrm{ng} / \mathrm{ml}$. Furthermore, selected parameters of lipid metabolism were analysed. Significantly higher average levels of TG in controls than in patients with CD $(p<0.01)$ were noticed $(99.2 \pm 25.4 \mathrm{mg} / \mathrm{dl}$ vs. $67.3 \pm 14.7 \mathrm{mg} / \mathrm{dl})$. Moreover, comparison of patients with $C D$, in the phase of exacerbation, with the control group revealed significantly higher levels of HDL cholesterol in the control group $(60.0 \pm 22.0 \mathrm{mg} / \mathrm{dl}$ vs. 41.6 $\pm 16.5 \mathrm{mg} / \mathrm{dl}$ ). Significantly higher concentrations of $\mathrm{HDL}$ in the control group than in UC patients in exacerbation, were observed $(60.0 \pm 22.0 \mathrm{mg} / \mathrm{dl}$ vs. $34.6 \pm 13.0$ $\mathrm{mg} / \mathrm{dl}) p<0.01$. The HDL/CHL ratio was significantly higher in the control group than in patients with UC in exacerbation phase: $0.351 \pm 0.061$ vs. $0.294 \pm 0.050$, $p<0.05$ (Tables I and II).

\section{Discussion}

Recent years have brought increased interest in adhesion molecules, which has greatly contributed to the explanation of inflammatory processes in the mucosa of the gastrointestinal tract. It appears that higher serum concentrations of ICAM, SVCAM, and SE-selectin in patients with IBD may indicate activation or damage of the vascular endothelial and possible migration process of inflammatory cells [11-16]. Our study showed no significantly higher values of marked adhesion molecules in patients with UC and CD in comparison to the control group. A statistically significant difference was found between VCAM average values in CD and UC patients in remission. We noticed that in this phase the standard variable VCAM was significantly lower in patients with CD than in patients with UC.

Table I. Summary of average values of the studied adhesion molecules in the studied groups of patients with IBD

\begin{tabular}{|c|c|c|c|c|c|c|c|c|c|c|c|}
\hline \multirow{3}{*}{$\begin{array}{l}\text { Adhesion } \\
\text { molecules } \\
{[\mathrm{ng} / \mathrm{ml}]}\end{array}$} & \multicolumn{4}{|c|}{$\mathrm{CD}(n=25)$} & \multicolumn{4}{|c|}{$\mathrm{UC}(n=15)$} & \multirow{2}{*}{\multicolumn{2}{|c|}{$\begin{array}{l}\text { Control } \\
(n=11)\end{array}$}} & \multirow[t]{3}{*}{$P$-value } \\
\hline & \multicolumn{2}{|c|}{$\begin{array}{l}\text { Exacerbation } \\
\qquad(n=16)\end{array}$} & \multicolumn{2}{|c|}{$\begin{array}{l}\text { Remission } \\
(n=9)\end{array}$} & \multicolumn{2}{|c|}{$\begin{array}{l}\text { Exacerbation } \\
\qquad(n=10)\end{array}$} & \multicolumn{2}{|c|}{$\begin{array}{l}\text { Remission } \\
\quad(n=5)\end{array}$} & & & \\
\hline & $x$ & SD & $x$ & SD & $x$ & SD & $x$ & SD & $x$ & SD & \\
\hline ICAM & 239.6 & 90.7 & 222.3 & 70.7 & 244.6 & 51.7 & 256.5 & 53.6 & 248.4 & 46.3 & \\
\hline VCAM & 889.5 & 273.4 & 762.9 & 189.9 & 825.1 & 168.5 & 942.5 & 225 & 880.8 & 175 & $<0.05$ \\
\hline E-selectin & 35.9 & 13.9 & 33.0 & 16.8 & 41.4 & 22.8 & 40.5 & 7.69 & 33.4 & 12.2 & \\
\hline
\end{tabular}

Table II. Summary of average values of lipid parameters in the group of patients with IBD

\begin{tabular}{|c|c|c|c|c|c|c|c|c|c|c|c|}
\hline \multirow{3}{*}{$\begin{array}{l}\text { Parameter } \\
{[\mathrm{mg} / \mathrm{dl}]}\end{array}$} & \multicolumn{4}{|c|}{$\mathrm{CD}(n=25)$} & \multicolumn{4}{|c|}{ UC $(n=15)$} & \multirow{2}{*}{\multicolumn{2}{|c|}{$\begin{array}{l}\text { Control } \\
(n=11)\end{array}$}} & \multirow[t]{3}{*}{$P$-value } \\
\hline & \multicolumn{2}{|c|}{$\begin{array}{l}\text { Exacerbation } \\
\quad(n=16)\end{array}$} & \multicolumn{2}{|c|}{$\begin{array}{c}\text { Remission } \\
(n=9)\end{array}$} & \multicolumn{2}{|c|}{$\begin{array}{l}\text { Exacerbation } \\
\quad(n=10)\end{array}$} & \multicolumn{2}{|c|}{$\begin{array}{l}\text { Remission } \\
(n=5)\end{array}$} & & & \\
\hline & $x$ & SD & $x$ & SD & $x$ & SD & $x$ & SD & $x$ & SD & \\
\hline $\mathrm{CHL}$ & 128.1 & 19.6 & 128.5 & 18.5 & 110.4 & 20.7 & 116.4 & 19.6 & 147.8 & 30.6 & $<0.05$ \\
\hline LDL & 74.3 & 12.16 & 63.0 & 13.9 & 62.2 & 19.2 & 49.6 & 19.6 & 70.8 & 17.6 & \\
\hline $\mathrm{HDL}$ & 41.6 & 16.46 & 51.0 & 18.9 & 34.6 & 13.0 & 46.4 & 24.3 & 60.0 & 22.0 & $<0.01$ \\
\hline $\mathrm{HDL} / \mathrm{CHL}$ & 0.318 & 0.100 & 0.395 & 0.144 & 0.294 & 0.05 & 0.338 & 0.097 & 0.351 & 0.061 & $<0.05$ \\
\hline TG & 79.7 & 21.0 & 67.3 & 14.7 & 95.7 & 59.3 & 100.0 & 39.1 & 99.2 & 25.4 & $<0.01$ \\
\hline
\end{tabular}


A similar study, but in a group of adult patients, was performed by Nielsen in 1994. He revealed increasing concentration of soluble form of ICAM-1 in correlation with activity of the inflammation process in the gastrointestinal tract [11]. Similar results were published a year later by Jones et al. They confirmed an increase of the concentrations of both ICAM and VCAM in active form of IBD in adults [12]. In contrast, Goke et al. did not confirm a correlation between higher than normal values of ICAM and VCAM and activity of inflammation [13]. In turn, Jones et al. examined the concentration of selected adhesion molecules. They showed higher VCAM levels in patients with UC in active phase than in patients in remission. However, they showed no such correlation in patients with CD and for ICAM in both groups [17]. Lazaris et al. demonstrated increased ICAM expression in vascular endothelium in areas of inflammation in patients with $U C$ and CD [14]. Vainer et al. found higher expression of ICAM in the inflamed fragment of the colonic mucosa (UC), but this relationship was not confirmed in patients with CD [18]. Adamska et al. assessed concentrations of ICAM and VCAM in children with CD and UC. Patients were divided into two groups, depending on the activity of the disease. The first group consisted of patients in remission, and the other group comprised patients with mild to moderate form of inflammatory bowel disease. Researchers showed higher levels of ICAM and lower VCAM in CD patients than in controls, but they did not show statistically significant values. What they revealed was significantly higher levels of ICAM in comparison to the comparative group of patients with UC. However, higher concentrations of VCAM in this group in comparison to the values set in the control group were not statistically significant. They also did not obtain statistically significant results of marked adhesion molecules depending on the stage of disease in patients with UC and from CD. Collected values of the analysed parameters in each group were similar [19].

Selectins contributes to recruitment of leukocytes to the place of inflammation. They take part in the initial phases of rolling of leukocytes to the blood vessel epithelium. In normal intestinal mucosa there is no expression of E-selectin; however, its concentration increases in a process of inflammation in intestines and correlates with the activity of inflammatory processes [20]. The influence of inflammatory mediators causes dislocation of selectins from the cytoplasm to the cell surface [21]. This process marks the beginning of rolling of leukocytes along the endothelial surface. Selectins on the surface of moving leukocytes bind to ligands presented on the surface of endothelial cells. That course contributes to the activation of leukocytes and leads to a change of conformation of the integrin receptors in the cell membrane of white blood cells. This is followed by squeezing of leukocytes between endothelial cells and their migration across basement membrane of capillary to the environment [2]. The consequences of this process have been confirmed by many clinical studies, inter alia by Lazaris et al. They demonstrated that increased expression of E-selectin on vascular endothelial cells of the colon is characteristic for active form of IBD [14]. Cellier et al. assessed both the expression of E-selectin in biopsies from the colonic mucosa and the concentration of the soluble sE-selectin in serum in adult patients with IBD. They showed a positive correlation between the expression of E-selectin in intestinal biopsies and the degree of activity of clinical, endoscopic, and histological IBD [16].

Based on our own results - no significantly higher values of marked adhesion molecules in patients with UC and CD compared to the control group were shown. Thus, it has not been found that the inflammatory process, in which adhesion molecules take part, contributes to an increased risk of developing atherosclerosis in these patients. Also, there were no increased values of examined lipid parameters, so there was no increased risk of developing atherosclerosis. On the contrary, it can be assumed that patients with IBD, in the phase of exacerbation, have lower risk of atherosclerosis as compared to the control group. This phenomenon may be a result of malnutrition, which occurs in periods of exacerbation. Despite the enormous progress made in medicine in recent years, impaired nutritional status is a very serious problem in patients with IBD. Nutrient deficiency, which is observed in patients with $\mathrm{IBD}$, produces a range of metabolic disorders and related complications, including anaemia or osteoporosis. To prevent these effects, therapeutic nutrition therapy should be included. The use of appropriate nutritional therapy, in addition to the pharmaceutical treatment, is very important in this group of patients. Appropriate nutritional therapy is included in the latest European Crohn's and Colitis Organisation (ECCO) guidelines as a therapeutic in CD and a supportive therapy of IBD. In children, in order to achieve remission, nutritional therapy is considered to be better tolerated and less aggravating than steroid therapy [22].

\section{Conclusions}

Although chronic inflammation includes both endothelial gastrointestinal and vascular endothelium, in children with IBD no risk factors of atherosclerosis were detected. Reduction of this risk was shown in patients in a stage of exacerbation of IBD, which might be a result of malnutrition typical for those diseases in children, especially in acute disease stage. 


\section{Acknowledgments}

This study was supported by ministerial grant Młody Naukowiec 2012/61MN.

\section{Conflict of interest}

The authors declare no conflict of interest.

\section{References}

1. Żerdziński M, Rychlik M, Partyka R. The selectins role in the development of inflamatory response. Diag Lab 2012; 48: 347-51.

2. Kwiatkowski P, Godlewski J, Śliwińska-Jewsiewicka A, et al. Cell adhesion molecules in the process of carcinogenesis and metastasis. Pol Ann Med 2009; 16: 128-37.

3. Mantur M, Wojszel J. Cell molecules and their participation in the process of inflammation and cancerogenesis. Pol Merk Lek 2008; 24: 177-80.

4. Kavey RE, Daniels RS, Lauer RM, et al. American Heart Association. Guidelines for primary prevention of atherosclerotic cardiovascular disease beginning in childhood. Circulation 2003; 107: 1562-6.

5. Berenson GS, Sririvan SR, Bao W, et al. Association between multiple cardiovascular risk factors and the early development of atherosclerosis. Bogalusa Heart Study. N Engl J Med 1998; 338: 1650-66.

6. Davis PH, Dawson JD, Riley WA, et al. Carotid intimal medial thickness is related to cardiovascular risk factors measured from childhood through middle age: the Muscatine Study. Circulation 2001; 104: 2815-9.

7. McMahan BW, Gidding SS, Malcom GT, et al. Pathobiological determinants of atherosclerosis in young risk scores are as sociated with early and advanced atherosclerosis. Pediatrics 2006; 118: 1447-55.

8. Magnussen CG, Venn A, Thomson R, et al. The association of pediatric low and high-density lipoprotein cholesterol dyslipidemia classification and change in dyslipidemia status with carotid intima-media thickness in adulthood. J Am Coll Cardiol 2009; 53: 860-9.

9. Głowińska-Olszewska B, Tołwińska J, Urban M. Interrelationship between endothelial dysfunction, ITM of the carotid arteries and adhesion molecules in obese hypertensive children and adolescents. Pediatr Endocrinol Diabetes Metab 2007; 13: 7-14.

10. Sappati Biyyani RS, Fahmy NM, Baum E, et al. Inflammatory bowel disease and coronary artery disease. Indian J Gastroenterol 2009; 28: 28-30.

11. Nielsen OH, Langholz E, Hendel J, Brynskov J. Circulating soluble intercellular adhesion molekule-1 (s ICAM) in active inflammatory bowel disease. Dig Dis Sci 1994; 39: 1918-23.

12. Jones SC, Banks RE, Haidar A, et al. Adhesion molecules in inflammatory bowel disease. Gut 1995; 36: 724-30.

13. Goke M, Hoffmann J C, Evers J, et al. Elevated serum concentrations of soluble selectin and immunoglobulin type adhesion molecules in patients with inflammatory bowel disease. J Gastroenterol 1997; 32: 480-6.

14. Lazaris AC, Dicoglou C, Tselein-Balaafouta S, et al. In situ expression of E-selectin and intercellular adhesion molecule-1 in chronic inflammatory diseases of the gastrointestinal tract. APMIS 1999; 107: 819-27.

15. Bhatti M, Chapman P, Peters M, et al. Visualizing E-selectin in the detection and evaluation of inflammatory bowel disease. Gut 1998; 43: 40-7.

16. Cellier C, Patey N, Fromont-Hankard G, et al. In situ endothelial cell adhesion molecule expression in ulcerative colitis. E-selectin in-situ expression correlates with clinical, endoscopic and histological activity and outcome. Eur J Gastroenterol Hepatol 1997; 9: 1197-203.

17. Jones SC, Banks RE, Haidar A, et al. Adhesion molecules in inflammatory bowel disease. Gut 1998; 36: 724-30.

18. Vainer B, Nieles $\mathrm{OH}$. Changed colonic profile of P-selectin, platelet-endothelial cell adhesion molekule-1 (PECAM-1), intercellular adhesion molekule-1 (ICAM-1), ICAM-2, and ICAM-3 in inflammatory bowel diseases. Clin Exp Immunol 2000; 121: 242-7.

19. Adamska I, Czerwionka-Szaflarska M, Kulwas A, et al. Adhesion molecules of immunoglobulin super family in children and youth with inflammatory bowel disease. Pol Merk Lek 2009; 26: 101-4.

20. Kazimierczak P, Wiśniewska-Jarosińska M, Drzewoski J. Adhesion molecules in inflammation bowel diseases. Gastroenterol Pol 2000; 7: 269-72.

21. Klintman D, Li X, Thorlacius H. Important role of P-selectin for leukocyte recruitment, hepatolocellular injury and apoptosis in endotoxemic mice. Clin Diagn Lab Immumol 2004; 11: 56-62.

22. Baczewska-Mazurkiewicz D, Rydzewska G. Nutritional problems in inflammatory bowel disease patients. Prz Gastroenterol 2011; 6: 69-77.

Received: 6.04.2016

Accepted: 17.08.2016 